\title{
Energy management in mobile robotics system based on biologically inspired honeybees behavior
}

\begin{abstract}
Operating multiple mobile robots while having finite amount of energy is one of limitation on doing a task in long term. In this work, a decentralized controller system based on inspired of swarm honeybees in foraging food's behavior has been developed and applied on the mobile robot. This system is simulated in Player/Stage. The algorithm is divided by working robot and foraging robot. The change of behaviors are based on remaining energy, which is defined as energy for worker and energy for forager. Mobile robots will be working while energy is enough and then, autonomously looking for energy source when battery in state of energy for forager. The information of position for power source energy that is getting from a forager robot will be shared with other mobile robots by local communication. In the player stage simulator, the communication process is done through UDP port. This experimental result shows that mobile robot is able to continuously work, forage, recharge and back to work by themselves without having intervention by human.
\end{abstract}

Keyword: Optimization; Power resource management; Swarm robotics 